\title{
Study on Detecting Location for Bus Rapid Transit with Signal Priority
}

\author{
Yangfan Zhou ${ }^{\mathrm{a}}$, Shunping Jia ${ }^{\mathrm{b}}$, Sijia Zhang ${ }^{\mathrm{c}}$, Wei Wei ${ }^{\mathrm{d}}$ \\ (MOE Key Laboratory for Urban Transportation Complex Systems Theory and Technology, Beijing \\ Jiaotong University, Beijing 100044, China) \\ a13114226@bjtu.edu.cn, b shpjia@bjtu.edu.cn, ${ }^{c} 14120927 @ b j t u . e d u . c n,{ }^{d} 13114223 @ b j t u . e d u . c n$,
}

Key words:Traffic engineering; Bus rapid transit; Signal priority; Detector; VISSIM

Abstract: Three signal priority scenarios were proposed for bus rapid transit(BRT) at isolated intersection based on early green and green extended strategies. The stopping time model of BRT at intersection was built considering signal phase status, detector location and its progression speed. Calculation results were compared with VISSIM simulation results, and the influence of detector location on the stopping time of BRT was analyzed under different progression speeds and cycle time. Results prove the effectiveness of model proposed in this paper. There is critical location for detector to minimize stopping time. When detector is closer to the stopping line than the critical location, stopping time decreases with detector's distance far away from stopping line. When it is beyond the critical location, stopping time is approaching to some stable value. Moreover, higher speed of bus causes longer stopping time and results in further critical location. Critical location almost has nothing to do with signal cycle time.

\section{Introduction}

Signal priority system is composed of signal control system and vehicle detecting system[1]. Signal priority strategies are the priority rules[2]. Early green and green extended are two common priority strategies[3]. Vehicle detection technology is the core of detecting system including automatic vehicle location[4], infrared detecting system[5], radio frequency identification devices[6], induction coil, and video[7]. Actually, detecting location has effect on the stopping time of priority vehicle at intersection which also influences priority effect.

Although there are studies on detecting location, some critical problems still have not been solved very well. Jeong[8] developed a method to ensure the optimal location of detector. However, simulation results were not identical with calculation results. Liu[9] holds the view that stopping time of bus has relationship with waiting time for green and queue clearance time. In his research, bus delay connected with detecting location was developed, but the relationship of them was achieved from simulation instead of the proposed model and lack of demonstration. Yan[10] got the conclusion that optimal detecting locations are $75 \mathrm{~m}$ and $150 \mathrm{~m}$ for early green and green extended respectively. However, these two priority strategies are conducted in compatible application and use the same detector in general. His research divided them into two scenarios and got two detecting locations which was not proper for the real.

It is necessary to explore the effect from detecting location on bus stopping time at intersection to inform optimal location under given priority strategies. Compared with the existing literatures, this paper put forward three priority scenarios clearly firstly. Calculating model is built then to release how the stopping time of bus changes with detecting location. Critical location for detector is achieved by the model and the results are closer to simulation. At last, influence factors of the relationship between them are analyzed. The results of changing regularity for stopping time with detecting location make sense to detecting system of BRT. 


\section{Priority scenarios for BRT}

Priority strategies are early green and green extended. Cycle time and minimum green times of non-priority phases are remained. Three specific scenarios are established according to where the extra green time of priority phase is from and whether early green is conducted or not once bus stops at intersection.

Scenario 1: the extra green time of priority phase is from all the other non-priority phases as shown in Fig. 1(a), and early green will not be conducted once bus stops.

Scenario 2: the extra green time of priority phase is from all the other non-priority phases as shown in Fig. 1(a), and early green is still conducted once bus stops.

Scenario 3: the extra green time of priority phase is just from the adjacent phases as shown in Fig. 1(b), and early green will not be conducted once bus stops.

Yellow time

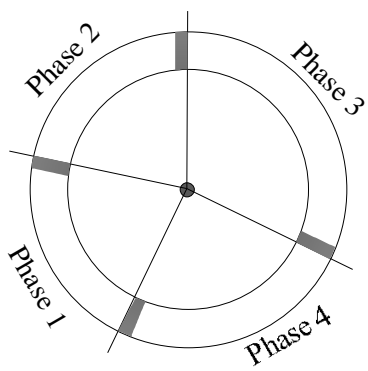

Normal phase
XX Minimum green time

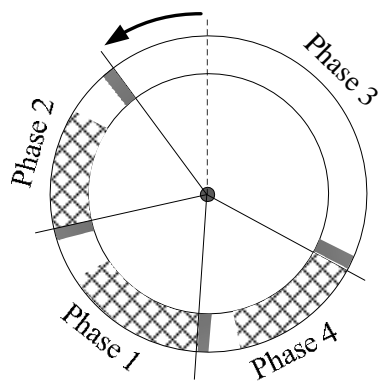

Early green
Priority phase: phase 3

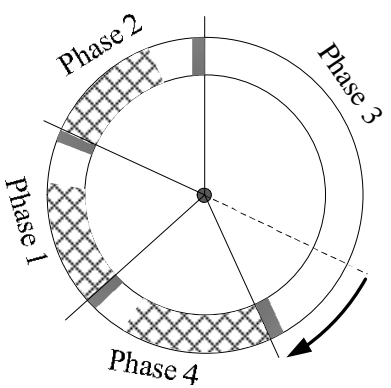

Green extended

(a) Priority phase diverts green time from non-priority phases (Scenario1, Scenario2)

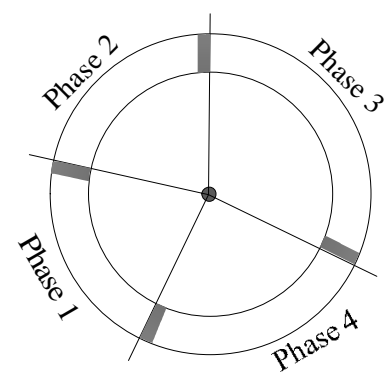

Normal phase

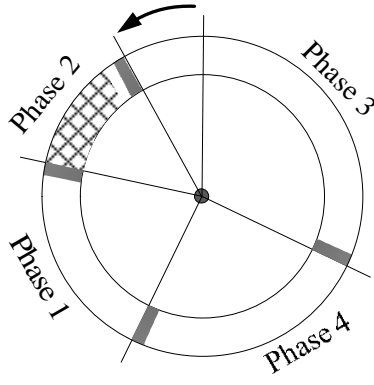

Early green

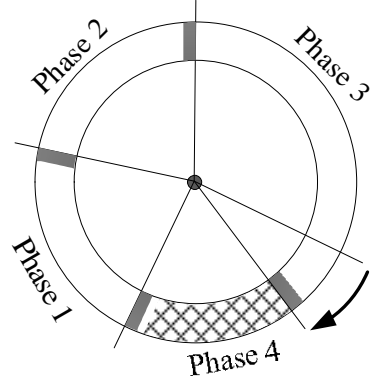

Green extended

(b) Priority phase diverts green time from its former and later phases (Scenario3)

Fig.1 Early green and green extended

\section{Stopping time of bus at intersection}

Assuming there is bus-only lane, detecting location is influenced by signal timing and progression speed of bus and has nothing to do with traffic flow. Moreover, there is no queue vehicle and ignore the station's impact. After detecting a bus in upstream, according to the distance from detector to stopping line and progression speed of bus, signal controller will judge whether conduct priority and the proper strategies considering signal condition at intersection. Based on this, let the signal is conducting at $t$ second of $k$ phase when detecting the bus. Stopping time model of bus is proposed for Scenario1, Scenario2 and Scenario3 by taking $k$ and $t$ as independent variables. Define variables and parameters as following.

$w$ : stopping time of bus at intersection, (s). $w_{1}$ is the stopping time in Scenario1.

$n: \quad$ sum of phase numbers.

$k: \quad$ phase number of signal when detecting bus, $k=1,2, \mathrm{~L}, n$.

$t$ : $\quad$ signal is conducting at $t$ second of $k$ phase when detecting bus, (s). 
$j: \quad$ priority phase number, $j=1,2, \mathrm{~L}, n$.

$i$ : $\quad$ phase number, $i=1,2, \mathrm{~L}, n$.

$C: \quad$ cycle time, (s).

$G_{i}$ : normal green time of phase $i,(\mathrm{~s})$.

$g_{i}: \quad$ minimum green time of phase $i,(\mathrm{~s})$.

$A_{i}: \quad$ yellow time of phase $i,(\mathrm{~s})$.

$l$ : $\quad$ distance from detector to stopping line, $(\mathrm{m})$.

$v$ : progression speed of bus between detector and stopping line, $(\mathrm{m} / \mathrm{s})$.

$\tau$ : deceleration, $\left(\mathrm{m} / \mathrm{s}^{2}\right)$.

$g_{\text {ext }}$ : maximum green extended time for priority, (s).

$g_{\text {ear }}:$ maximum early green time for priority, (s);

For Scenario1, according to the relationship between the phase when detecting bus and the priority phase, there are three situations, $k<j, k>j$ and $k=j$, need to be discussed

(1) If $k<j$, there are three stopping times according to the time when bus arrives at the stopping line.

1) Even conducting early green, bus still arrives at the stopping line before green light. It will stop and wait for the green light. So $0 \leq \frac{l}{v}<\sum_{i=k}^{j-1}\left(\mathrm{~g}_{i}+A_{i}\right)-t$.

$$
w_{1}(k<j, t)=\sum_{i=k}^{j-1}\left(G_{i}+A_{i}\right)-t-\frac{l}{v}-\frac{v}{2 \tau}
$$

2) Bus can drive through the intersection in green time without stop. So $\sum_{i=k}^{j-1}\left(\mathrm{~g}_{i}+A_{i}\right)-t \leq \frac{l}{v}<\sum_{i=k}^{j-1}\left(G_{i}+A_{i}\right)+G_{j}+g_{e x t}-t$

$$
w_{1}(k<j, t)=0
$$

3) Even conducting green extended, bus still cannot go through the stopping line before green time finishes. It has to wait for the next green time. So $\sum_{i=k}^{j-1}\left(G_{i}+A_{i}\right)+G_{j}+g_{\text {ext }}-t \leq \frac{l}{v} \leq C$.

$$
w_{1}(k<j, t)=C+\sum_{i=k}^{j-1}\left(G_{i}+A_{i}\right)-t-\frac{l}{v}-\frac{v}{2 \tau}
$$

(2) If $k>j$, there are three stopping times as following.

1) Even conducting early green, bus still arrives at the stopping line before green light. So $0 \leq \frac{l}{v}<\sum_{\substack{i=1 \\(i \neq j\llcorner k-1)}}^{n}\left(g_{i}+A_{i}\right)-t$.

$$
w_{1}(k>j, t)=\sum_{\substack{i=1 \\(i \neq j\llcorner k-1)}}^{n}\left(G_{j}+A_{i}\right)-t-\frac{l}{v}-\frac{v}{2 \tau}
$$

2) Bus can drive through the intersection in green time without stop. So $\sum_{\substack{i=1 \\(i \neq j L \\ k-1)}}^{n}\left(\mathrm{~g}_{i}+A_{i}\right)-t \leq \frac{l}{v}<\sum_{\substack{i=1 \\(i \neq j \mathrm{~L} k-1)}}^{n}\left(G_{i}+A_{i}\right)+G_{j}+g_{\text {ext }}-t$.

$$
w_{1}(k>j, t)=0
$$

3) Even conducting green extended, bus still cannot go through the stopping line before green time finishes. So $\sum_{\substack{i=1 \\(i \neq j<k-1)}}^{n}\left(G_{i}+A_{i}\right)+G_{j}+g_{\text {ext }}-t \leq \frac{l}{v} \leq C$. 


$$
w_{1}(k>j, t)=C+\sum_{\substack{i=1 \\(i \neq j\llcorner k-1)}}^{n}\left(G_{j}+A_{i}\right)-t-\frac{l}{v}-\frac{v}{2 \tau}
$$

(3) If $k=j$, there are two stopping times because early green cannot be conducted.

1) Bus can drive through the intersection in green time without stop. So $0 \leq \frac{l}{v}<G_{j}+g_{\text {ext }}-t$.

$$
w_{1}(k=j, t)=0
$$

2) Even conducting green extended, bus still cannot go through the stopping line before green time finishes. So $\frac{l}{v} \leq G_{j}+g_{\text {ext }}-t \leq C$.

$$
w_{1}(k=j, t)=C-t-\frac{l}{v}-\frac{v}{2 \tau}
$$

It is similar for Scenario2 and Scenario3 to get the stopping time of bus. Let $\alpha_{1}, \alpha_{2}$ and $\alpha_{3}$ are $0-1$ variables. If they equal zero, bus doesn't stop at the intersection, while equal one, bus stops. Let $\beta_{1}$ and $\beta_{2}$ are $0-1$ variables. If they equal zero, green time has not came when bus arrives, while equal one, green time has finished and bus needs to wait for the next green light to pass. $a$ is 0-1 variable. $a=0$ in Scenario1, $a=1$ in Scenario2, and $a=0$ in Scenario3. $b$ is also 0-1 variable. $b=0$ in Scenario1, $b=0$ in Scenario2, and $b=1$ in Scenario3. Stopping time in three scenarios under various situations can be expressed by function (9).

$$
w(k, t)= \begin{cases}\alpha_{1} \times\left\{\beta_{1} \times\left[C+a\left(\sum_{i=k}^{j-1}\left(G_{i}-g_{i}\right)-g_{\text {ear }}\right)\right]+\sum_{i=k}^{j-1}\left[a g_{i}+(1-a) G_{i}+A_{i}\right]-t-\frac{l}{v}-\frac{v}{2 \tau}\right\} & , k<j \\ \alpha_{2} \times\left\{\beta_{2} \times\left[C+a\left(\sum_{\substack{i=1 \\(i \neq j L k-1)}}^{n}\left(G_{i}-g_{i}\right)-g_{\text {ear }}\right)\right]+\sum_{\substack{i=1 \\ i \neq j \mathrm{~L} k-1)}}^{n}\left[a g_{i}+(1-a) G_{j}+A_{i}\right]-t-\frac{l}{v}-\frac{v}{2 \tau}\right\} & , k>j \\ \alpha_{3} \times\left(C-t-\frac{l}{v}-a g_{\text {ear }}-\frac{v}{2 \tau}\right) & , k=j\end{cases}
$$

where,

$$
\begin{aligned}
& \alpha_{1}=\left\{\begin{array}{l}
0, \sum_{i=k}^{j-1}\left[b G_{i}+(1-b) g_{i}+A_{i}\right]-b g_{\text {ear }}-t \leq \frac{l}{v}<\sum_{i=k}^{j-1}\left(G_{i}+A_{i}\right)+G_{j}+g_{\text {ext }}-t \\
1, \text { else }
\end{array}\right. \\
& \alpha_{2}=\left\{\begin{array}{l}
0, \quad \sum_{\substack{i=1 \\
(i \neq j \mathrm{~L} k-1)}}^{n}\left[b G_{i}+(1-b) \mathrm{g}_{i}+A_{i}\right]-b g_{\text {ear }}-t \leq \frac{l}{v}<\sum_{\substack{i=1 \\
(i \neq \mathrm{L} k \mathrm{k}-1)}}^{n}\left(G_{i}+A_{i}\right)+G_{j}+g_{\text {ext }}-t \\
1, \text { else }
\end{array}\right. \\
& \alpha_{3}=\left\{\begin{array}{l}
0,0 \leq \frac{l}{v}<G_{j}+g_{\text {ext }}-t \\
1, \text { else }
\end{array}\right. \\
& \beta_{1}=\left\{\begin{array}{l}
0,0 \leq \frac{l}{v}<\sum_{i=k}^{j-1}\left[b G_{i}+(1-b) \mathrm{g}_{i}+A_{i}\right]-b g_{\text {ear }}-t \\
1, \text { else }
\end{array}\right. \\
& \beta_{2}=\left\{\begin{array}{l}
0,0 \leq \frac{l}{v}<\sum_{\substack{i=1 \\
(i \neq j\llcorner k-1)}}^{n}\left[b G_{i}+(1-b) \mathrm{g}_{i}+A_{i}\right]-b g_{\text {ear }}-t \\
1, \text { else }
\end{array}\right.
\end{aligned}
$$

It is a random probability event to detect bus if ignoring timetable. Let $W$ is the expectation 
value of stopping time. So,

$$
W=\mathrm{E}(w)=\sum_{k, t} \frac{w(k, t)}{C}
$$

\section{Case study}

Take a four phases intersection as case, shown in Fig. 2. There are bus-only lanes for east-west directions. Signal timing is presented in table 1.

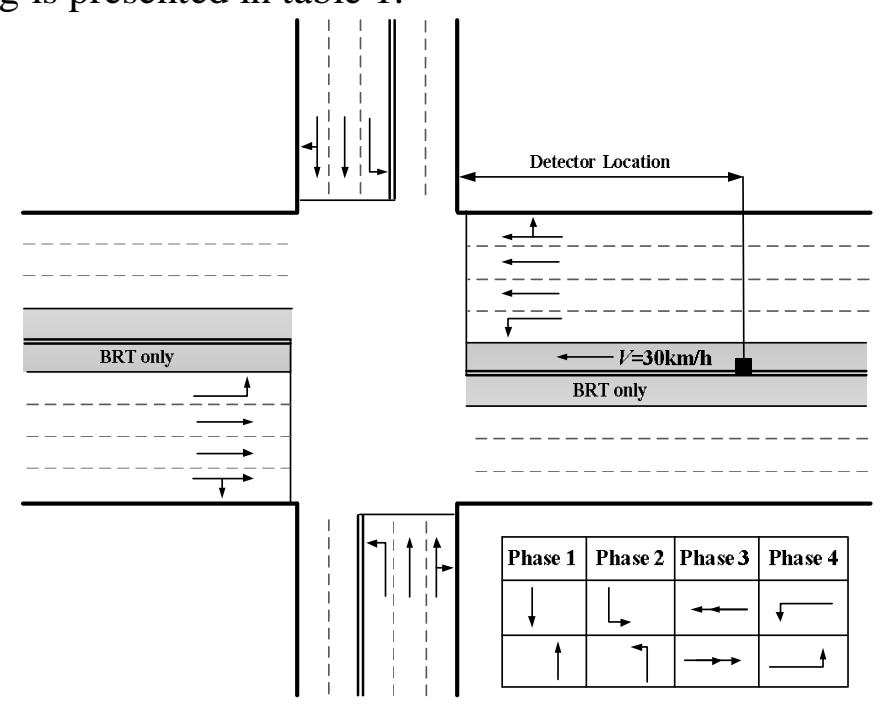

Fig. 2 Phase sequence of the intersection

Table 1 Signal parameters of the intersection (unit: second)

\begin{tabular}{ccccc}
\hline Cycle time & Phase & Minimum green time & Normal green time & Yellow time \\
\hline \multirow{3}{*}{120} & 1 & 27 & 32 & 3 \\
& 2 & 17 & 22 & 3 \\
& 3(Priority phase) & 32 & 32 & 3 \\
& 4 & 17 & 22 & 3 \\
\hline
\end{tabular}

Results and analysis. Let $l=50,60, \ldots 300(\mathrm{~m}), g_{\text {ext }}=15 \mathrm{~s}$, and $g_{\text {ear }}=15 \mathrm{~s}$. Curves of stopping time changing with detecting location in three scenarios are shown in Fig. 3.

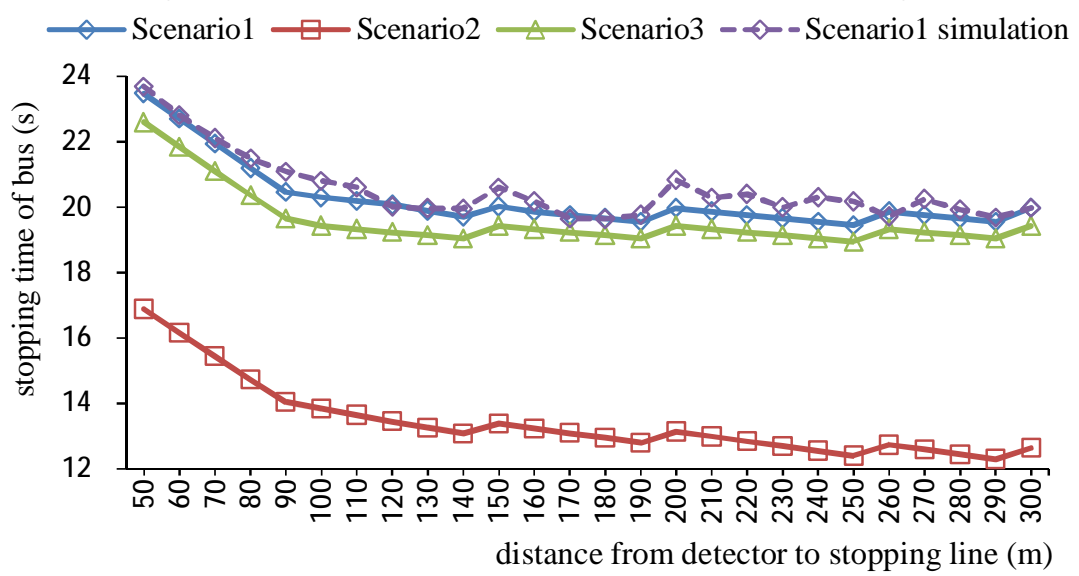

Fig. 3 Stopping time of bus varies from detecting location in three scenarios

It is obvious from Fig. 3 that stopping time of bus decreases firstly along with detector far away from the stopping line, and then tends to be a stable value after the detector is placed beyond some location. Thus stopping time of bus will be relative low when the distance from stopping line to detecting location is more than some value which is called critical location in this paper. In these three scenarios, the decreases of stopping time drop from $3.5 \%$ to $0.8 \%, 4.7 \%$ to $1.4 \%, 3.5 \%$ to $1.1 \%$ respectively at $90 \mathrm{~m}$ of detector. Therefore, $90 \mathrm{~m}$ is the critical detecting location for stopping 
time. It is better to settle detection system upstream beyond $90 \mathrm{~m}$ to get a lower stopping time of bus. Actually, considering the bus stop location, passenger convenience, progression speed volatility, detector is not proper to settle too far away from intersection. With overall consideration, $90 \mathrm{~m} \sim 150 \mathrm{~m}$ is the suggestive location of detector.

For demonstrating the correctness of model proposed in this paper, simulation is conducted in virtue of VISSIM. The same actuated signal control plan with Scenario1 is designed through VisVAP. Curve is shown as dashed line in Fig. 3. Simulation results are almost the same with calculation results. The average value of their differences is just $0.31 \mathrm{~s}$ and the variance is $0.8 \mathrm{~s}^{2}$, which proves the model is effective.

Influence of progression speed of bus. In Scenario1, let the progression speed of bus $v=10,20$, $30,40,50(\mathrm{~km} / \mathrm{h})$. The relationships between stopping time and detecting location in various speeds are calculated and shown in Fig. 4.

When bus is quite slow like $v=10 \mathrm{~km} / \mathrm{h}$, stopping time is about $21 \mathrm{~s}$ and almost doesn't influenced by detecting location. As the speed developing, the critical location is becoming farther. Critical location is $70 \mathrm{~m}$ when $v=20 \mathrm{~km} / \mathrm{h}, 90 \mathrm{~m}$ when $v=30 \mathrm{~km} / \mathrm{h}, 130 \mathrm{~m}$ when $v=40 \mathrm{~km} / \mathrm{h}$, and $160 \mathrm{~m}$ when $v=50 \mathrm{~km} / \mathrm{h}$, shown as the black marks in figure. The average stopping time is positive correlation with speed as detector is closer to intersection than the critical location. While if the detector is beyond than the critical location, stopping time tends to be a stable value and this value is smaller as speed larger.

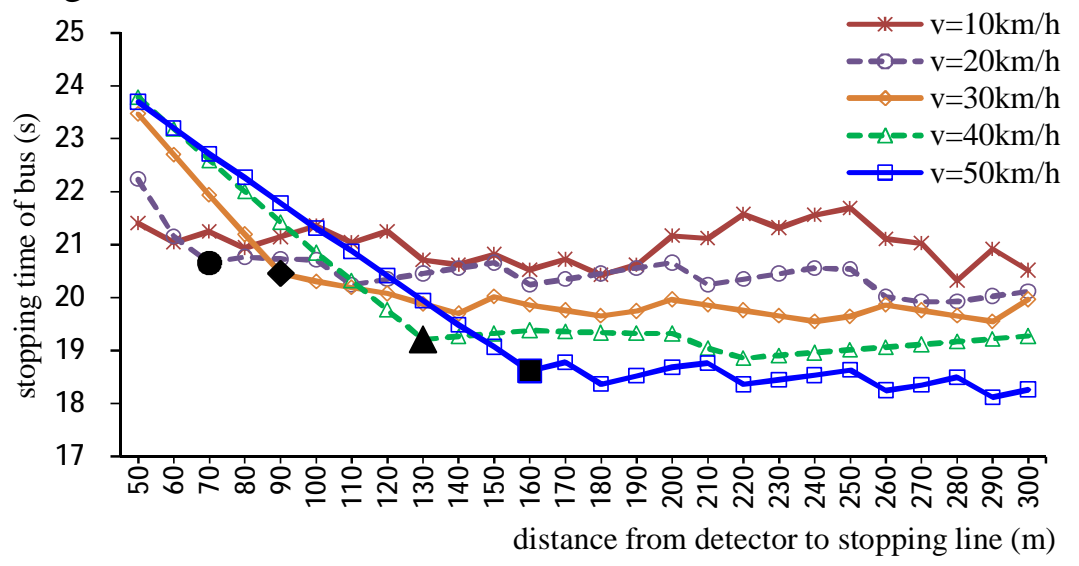

Fig. 4 Stopping time of BRT varies from detecting location under different speed

Influence of cycle time. In Scenario1, let the cycle time $C=80,100,120,140,160(\mathrm{~s})$. Green time of each phase changes with cycle time by an interval of $5 \mathrm{~s}$. The priority phase's green time $G_{3}=22,27,32,37,42(\mathrm{~s})$ accordingly. The relationships between stopping time and detecting location in various cycle times are calculated and shown in Fig. 5.

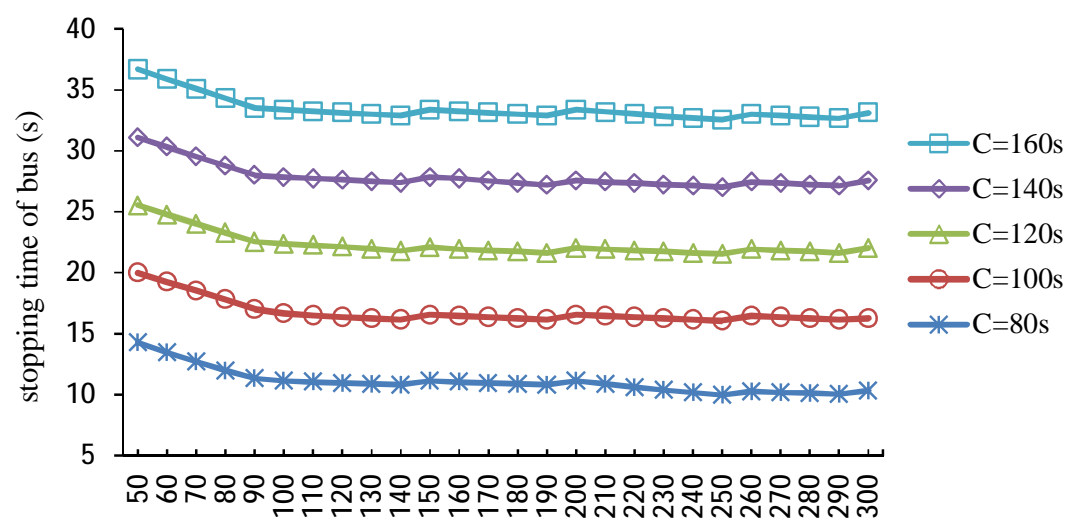

distance from detector to stopping line $(\mathrm{m})$

Fig. 5 Stopping time of BRT varies from detecting location under different cycle time

It can be concluded that the critical locations are all $90 \mathrm{~m}$ in different cycle times. They are a 
group of parallel curves with the same separation distance by $5 \mathrm{~s} \sim 6 \mathrm{~s}$ which equals the difference of green times of priority phase. Therefore, cycle time just influences stopping time value, while doesn't effect on their relationship between stopping time and detecting location. In addition, the critical location is the same in various cycle times.

\section{Conclusions}

Signal priority is helpful to improve efficiency and travel speed of BRT. Detecting location has effect on its stopping time at intersection. This paper developed a model to describe the relationship between stopping time of bus and detecting location. Simulation results proved its correctness and effectiveness. The results show that there is critical location for detector to make stopping time quite small and this critical location is influenced by bus speed. Some further researches can focus on other different priority scenarios.

\section{Acknowledgements}

This research was funded by the Fundamental Research Funds for the Central Universities (2015YJS090), the National Basic Research Program of China (No. 2012CB725406) and the National Natural Science Foundation of China (71131001).

The authors also thank reviewers and editors for their suggestions to improve this paper.

\section{References:}

[1] M. A. C. Aleman. Evaluation of Bus Priority Strategies for BRT Operations[D]. Boston: Massachusetts Institute of Technology, 2013.

[2] S. Kim, M. Park, K. S. Chon. Bus Signal Priority Strategies for Multi-directional Bus Routes[J]. Ksce Journal of Civil Engineering, 2012, 16(5):855-861.

[3] P. J. Zhang, C. Liao, Y. Z. Yang, et al. Study on the Signal Priority of BRT by the Simulations Based on the Cellular Automata Model[J]. Journal of Transportation Systems Engineering and Information Technology, 2011, 11(S1):61-67.

[4] D. H. Wang, H. Zhu, Y. M. Bie, et al. Bus Signal Priority Method at Arterial Signal Progression[J]. Journal of Southeast University (Natural Science Edition), 2011, 41(4):859-865.

[5] W. J. Ma, Y. Liu, X. G. Yang. A Dynamic Programming Approach for Optimal Signal Priority Control Upon Multiple High-Frequency Bus Requests[J]. Journal of Intelligent Transportation Systems, 2013, 17(4):282-293.

[6] Z. Tan, Z. Y. Mei, Z. Y. Huang, et al. Optimal Cycle Model Based on Active Transit Signal Priority Strategies with Artery Coordination[J]. Journal of Highway and Transportation Research and Development, 2012, 29(7):104-110.

[7] H. F. Xu, M. M. Zheng. Impact of Bus-Only Lane Location on the Development and Performance of the Logic Rule-Based Bus Rapid Transit Signal Priority[J]. Journal of Transportation Engineering, 2014, 138(3):293-314.

[8] Y. Jeong, Y. Kim. Study on the Optimal Detector Location for the Active Bus Signal Priority of the Median Bus Lane[C]// 17th ITS World Congress. 2010.

[9] H. C. Liu, A. Skabardonis, W. B. Zhang, et al. Optimal Detector Location for Bus Signal Priority[J]. Transportation Research Record: Journal of the Transportation Research Board, 2004(1864):144-150.

[10]F. Yan, K. P. Li, J. Sun. Detector Location for Transit Signal Priority at Intersection with Countdown Signals[J]. Journal of Transportation Information and Safety, 2009, 27(6):79-83. 